\title{
Is the Medium the Message?: \\ Examining Transactions Conducted via Text in Comparison with Traditional Virtual Reference Methods
}

Tara Mawhinney, McGill University

Svetlana Kochkina, McGill University

\begin{abstract}
Texting is a widespread mode of communication and libraries are experimenting with it to interact with users. This article presents the results of a study on virtual reference service. The authors seek to determine whether or not the texting service is filling a different information need than chat and email by examining the level of difficulty and the topic of questions using these different methods of communication. Findings indicate a marked difference between the type and complexity of questions sent via texts and traditional forms of virtual reference, which suggests that texting fills a complimentary role in relation to other traditional virtual reference communication channels used within libraries.
\end{abstract}

Keywords: academic libraries, virtual reference, text reference

\section{Introduction}

Marshall McLuhan, a famous Canadian public intellectual, coined the phrase "the medium is the message" (1964, p. 7) in the early 1960s. The phrase has just as much importance as ever today when we consider the extent to which different forms of media shape the messages that they transmit. In a context where people have ever-increasing methods of communication from which to choose, does the method they choose impact what they say? To what extent does the medium of communication determine the message? Within the context of an academic library offering a variety of methods of communication to its users, what is the relationship between the method a user chooses and what the user says?

McGill University Library is a large academic library in Canada, serving a population of 40,000 students, which has provided virtual reference service via chat and email since 2006 . The 
virtual reference service continues to be popular with an overall trend showing a steady increase in usage with a total of 6,528 questions received in 2016-2017 academic year, the highest yet recorded. The library uses QuestionPoint, an OCLC product, as the virtual reference software. The service is not provided on a consortial basis but rather is staffed primarily by public services librarians within the institution and second-year masters students from the McGill School of Information Studies. In 2016, in an effort to ensure service excellence and responsiveness to user needs, texting was introduced on a pilot project basis as an additional means of communication between users and the library.

Texting is a popular medium of communication, especially among students. A Pew Research Center study on smartphone use reports that texting is the feature of smartphones that is the most frequently used by respondents, with younger respondents being the most avid users (2015). One of the rationales for introducing this new service at McGill was that it is an urban campus located in the Montreal metropolitan area where students are often on the move and outside of the university's wi-fi zones, making texting a very convenient alternative to traditional virtual reference in the form of email and chat.

In the context of assessing this new service, the study aims to discover if users contact the library via the texting service for specific types of questions, different from the kinds of questions they send through other methods of virtual reference. Specifically, the authors seek to determine whether or not the texting service is filling a different information need than other venues by examining the level of difficulty and the topic of questions using these different methods of communication by answering the following research questions:

1. Does the level of complexity of the questions arriving via virtual reference vary, depending on the choice of medium through which they are sent?

2. Does the type/topic of questions vary, depending on the choice of medium through which they are sent?

In addition, the authors compare the results of this analysis with their previous qualitative study of chat and email transactions dating from 2014 (Côté, Kochkina, \& Mawhinney, 2016) to 
determine if there have been changes over time to the level of complexity and type of questions received via chat and email.

\section{Literature Review}

Some previous literature exists comparing texting with more traditional types of virtual reference service, namely chat and email. Gervasio alludes to the environment from which a text transaction could derive noting that "patrons text librarians from physical locations in the wider world: from a bus stop, a grocery store, a remote corner of the library stacks" (2014, p. 46). If users are contacting the library from these disparate locations, one can infer that the nature and level of difficulty of text transactions could be very different from those of other types of virtual reference. Several researchers suggest that texting transactions are very different in the type and level of questions from other methods of virtual reference. Breitbach and Prieto report that in comparison with chat and email, the percentage of texting questions of a complex nature is much lower (2012, p. 191). Gervasio finds questions in texting to be quick-answer type questions and that most transactions do not consist of very much back and forth interaction (2014, p. 54). She claims that users "self-select which tool is best adapted to their query" (p. 54). Peters, from his experience answering text queries via My Info Quest, a mobile reference collaborative with over 50 library organizations supplying the staffing, claims most texting transactions do not require the "Great Chain of Reference Questioning" since they are often of a directional or opinion-seeking in nature (2010, p. 95). Breitbach and Prieto also report that texting is mostly of the quick answer variety, claiming that patrons are "self-regulating" the type of questions they ask in a texting environment (2012, p. 194). Similarly, Luo states that users in a texting environment often want easy and quick answers (2011, p. 489). Vecchione and Ruppel's advice to service providers is to: "respond as simply as possible to a reference question" in a texting environment (2012, p. 367).

However, not all research is unanimous in finding that certain types of questions lend themselves to the texting format rather than other venues. Some literature recommends treating texting like a venue no different from existing venues for virtual reference service. As Collard, Whatley, \& Pearce state:

In contrast to some of the assumptions in the library literature that users would prefer this service medium only for short transactions, our data indicate that reference service via SMS 
aligns closely with the services we offer elsewhere and that the same guiding reference philosophies we employ in our other services yield higher levels of user satisfactions... it is our analysis of SMS transcripts during the past couple of years that has permitted us to fully embrace a venue-blind approach to service. (2011, p. 382)

Likewise, Vardeman and Barba's study of three years of data from texting transactions from a library at a large, public university shows that although questions via texting tend to be short, they resemble questions asked through other virtual and in person mediums (2014, p. 173). These studies suggest that a "keeping it short" approach is not needed and that a better approach would be a "venue-blind" one, as suggested by Pearce et al., based on their findings reported in several publications (Pearce, 2010; Pearce, Collard, \& Whatley, 2010a; Pearce, Collard, \& Whatley, 2010b; Collard et al., 2011).

Another issue arising from the previous literature is dichotomy between reported high use of texting in everyday life (Pew Research Center, 2015) and its low use in libraries, as reported in previous literature (Vardeman \& Barba, 2014, p. 175; Cole \& Krkoska, 2011, p. 6; Breitbach \& Prieto, 2012, p. 193), questioning the long-term viability of texting as a medium for virtual reference. Vardeman and Barba, attribute low use to several factors: difficulties with using texting, marketing, and users' preference for chat over texting (2014, p. 178). Breitbach and Prieto, reporting on their pilot project of texting at a large library that is part of a one of the two public university systems in California, state that texting use was low, despite similar levels of promotion as when chat reference was piloted (2012, p. 193). They speculate that low use may have been partly due to long response times resulting from technical difficulties and limited service hours (p. 195). Perhaps the low use indicates that users want to be able to ask more complex questions than texting enables. Another possibility is that texting requires a different kind of promotion that other forms of virtual reference. Or perhaps users simply prefer chat to texting for virtual reference. If that is the case, this would mirror findings from the retail sector, reporting live chat to have the highest satisfaction ratings among different customer service channels with a 73\% satisfaction rate compared to $41 \%$ for texting, falling as the lowest-rated customer service touch point, ranking even lower than satisfaction with customer service by phone (Charlton, 2013). Likewise, previous 
research from two US university libraries indicate that students rank chat reference more highly than other virtual reference methods, including texting (Chow \& Croxton, 2014, p. 318).

Yet, despite chat being more popular and authors reporting low use of texting at the current time, is this service nonetheless worthwhile? Chow and Croxton state that although usage of texting may be low at the current time, it "may soon become a more prevalent reference medium, regardless of the type of question" (2012, p. 248). They specify that younger participants rank texting more highly than older ones and that given that younger users will continue to be heavy text users, libraries should be ready to meet the need of these users via texting services (pp. 25859). Likewise, a systematic review on virtual reference suggests that texting is likely to become increasingly important in the future (Matteson, Salamon, \& Brewster, 2011, p. 185), as does other previous literature (Ruppel \& Vecchione, 2012, p. 443).

Interestingly, Vardeman and Barba report no marked decrease in chat and email transactions when texting was implemented (2014, p. 175). These findings suggest that texting is filling a different information need than other virtual reference channels. Not only that but other research indicates that texting reaches a different user group than other channels (Vecchione \& Ruppel, 2012, p. 369). These factors alone could justify implementation of a texting service.

As noted by Vecchione and Ruppel, the existing literature published up until 2012 shows that there is not adequate research on what people are asking in the texting environment (2012, p. 358). Texting is a relatively new service, and as noted by Vardeman and Barba, much of the literature on texting in libraries reports on anecdotal evidence from a given library's first experiences with texting (2014, p. 164), rather than reporting on results from research studies. The current study will contribute to the limited existing literature by reporting on a more rigorous examination of the question of whether or not texting is filling a distinct information need.

\section{Methods}

The authors hypothesized that the medium of communication affects the type and complexity of questions, i.e. users chose to send certain types of questions either through chat, email, or text, depending on the nature of the question they are asking. To test the hypothesis, a qualitative 
analysis of a sample of chat, email, and text transactions was conducted in order to discover and compare the levels of complexity of the questions and the distribution of questions by topic. The sample period was from June $4^{\text {th }}, 2016$ to June $4^{\text {th }}, 2017$, a time that corresponded to the texting pilot project. The sample consisted of 130 texts, the total number of transactions conducted via text in the pilot year, and 130 each of chats and emails, selected using systematic random sampling from the transactions that occurred in the same 12 months of 2016-2017. During the pilot period, the total number of text messages received from users in QuestionPoint was 324. The software does not allow for threading of text messages, so received individual texts were collated into meaningful transactions. After the transactions were collated, as well as after blank and duplicate questions were removed, the total number of text transactions amounted to 130 . The total number of chats accepted by the library for the same period was 5,392. Due to technical issues, it was possible to retrieve only 3,807 chats from the system, all of which were from the entire duration of the sampling period. The sampling interval of 29 was used to obtain 130 chats to be included in the analyzed sample. In the same time period, 3,387 emails were received in the virtual reference software. For the emails, the sampling interval used was 26.

The transactions were divided between two librarians who coded them in an Excel spreadsheet. To ensure consistency of the analysis, a codebook was used by the authors. To enhance inter-coder reliability, previously coded questions were randomly sampled and re-coded by the other coder. For each question, the authors noted the data regarding reference transactions that was automatically collected by the software, such as means of communication (text, chat, or email). The authors analyzed the content of the transactions to determine question type/topic and the level of complexity (basic, intermediate, advanced). The definitions of each level of complexity, similar to the previous study (Côté, Kochkina, \& Mawhinney, 2016), were aligned with those used for gathering statistics of in-person reference transactions at McGill Library as follows:

- basic: responds to a simple question using library information sources (catalogue, website, ready reference);

- intermediate: assists users with intermediate-level questions or support, may require use of several information sources, and often involves user instruction; 
- advanced: responds to a user's question using advanced expertise in the service area. Interactions are often multi-faceted and/or inter-disciplinary and subject specialists may need to be consulted.

Coders analyzed whether or not a question was referred. A referred question was defined as a case where a user was directed to another staff member or service unit to be given a response to their query. Referred questions included both those where the staff member forwarded the user's question along to an appropriate individual or service unit and those where the user was asked to contact an appropriate individual or service unit him or herself.

The transactions were analyzed and coded using a modified coding scheme (see Appendix 1) that was initially developed for the qualitative study of chat and email transactions dating from 2014 (Côté, Kochkina, \& Mawhinney, 2016). The same coding scheme was used in both studies to ensure comparability of the results. The comparison was done to determine if there had been changes over time to the level of complexity and type of questions received via chat and email. The only modification in the scheme consisted of further breaking down the "McGill Library Services" category of questions into three subcategories: policy, hours, and other service-related questions, while leaving all loan questions in the loan category. This subdivision was done keeping in mind that previous studies of texting, as mentioned earlier, reveal high percentages of quickanswer type questions, which we presumed would often be related to library hours and policies. However, this modification did not prevent comparison of the results with the previous study as these three subcategories can be combined into one.

After the data was recorded, the transactions were analyzed by the authors, using the coding scheme described above to compare:

- The level of complexity of questions received via text vs. via email and chat.

- The type/topic of questions received via text vs. via email and chat. The question types/topics were compared using both new subcategories (policy, hours, and other servicerelated questions) and a broader "McGill Library Services" category.

- The number of question referred to other librarian or other unit received via text vs. via email and chat. 
The results of the analysis for the chat and email transactions from the current study were compared with the results of the previous qualitative study of chat and email transactions from 2014. The goal was to identify if there were any trends or changes with regard to: distribution of questions by level of question difficulty and types/topics, as well as in the number of referrals.

\section{Findings}

\section{Question Level}

The findings confirm the authors' hypothesis that the medium of communication affects the complexity and type of questions users ask. There is a marked difference in the level of complexity between texts and chat/emails and also in the type/topic of questions, as outlined below. The analysis of the levels of complexity of the questions demonstrates that users primarily chose to send basic rather than intermediate-level questions through text, with $81 \%$ of transactions being of the basic level. This compares to just $43 \%$ of chat/email transactions being basic (see Figures 1 and 2).

\section{Figure 1. Question Level: Text}

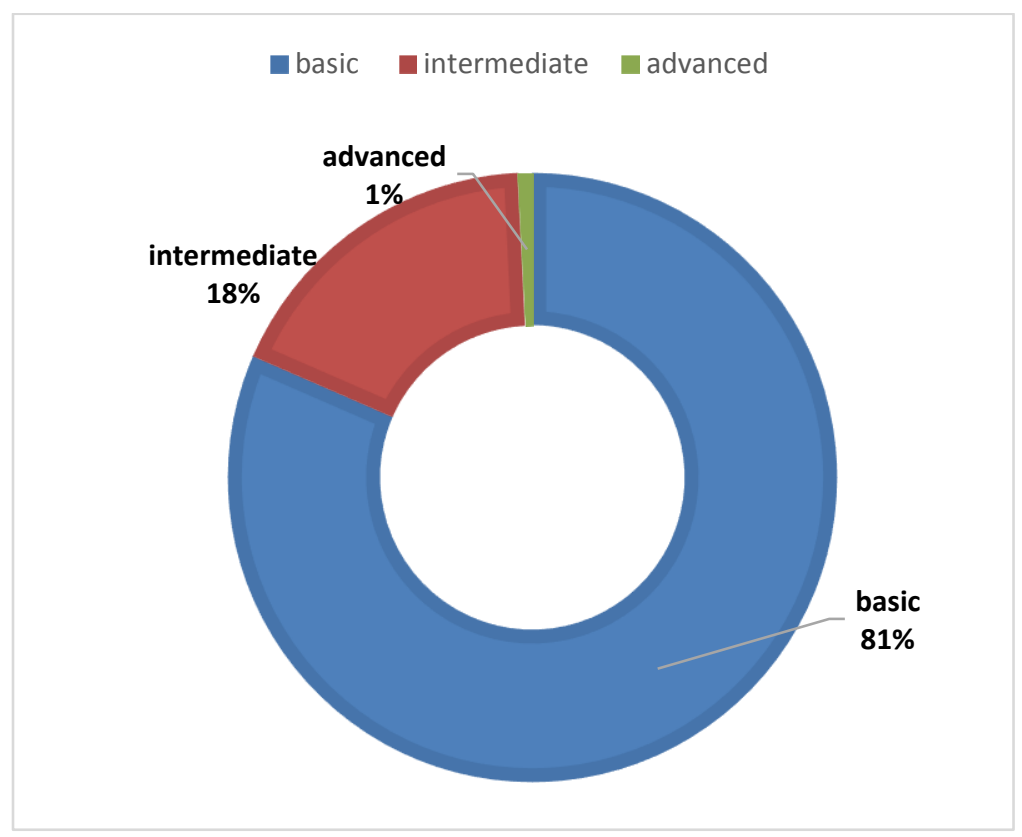


Figure 2. Question Level: Chat \& Email

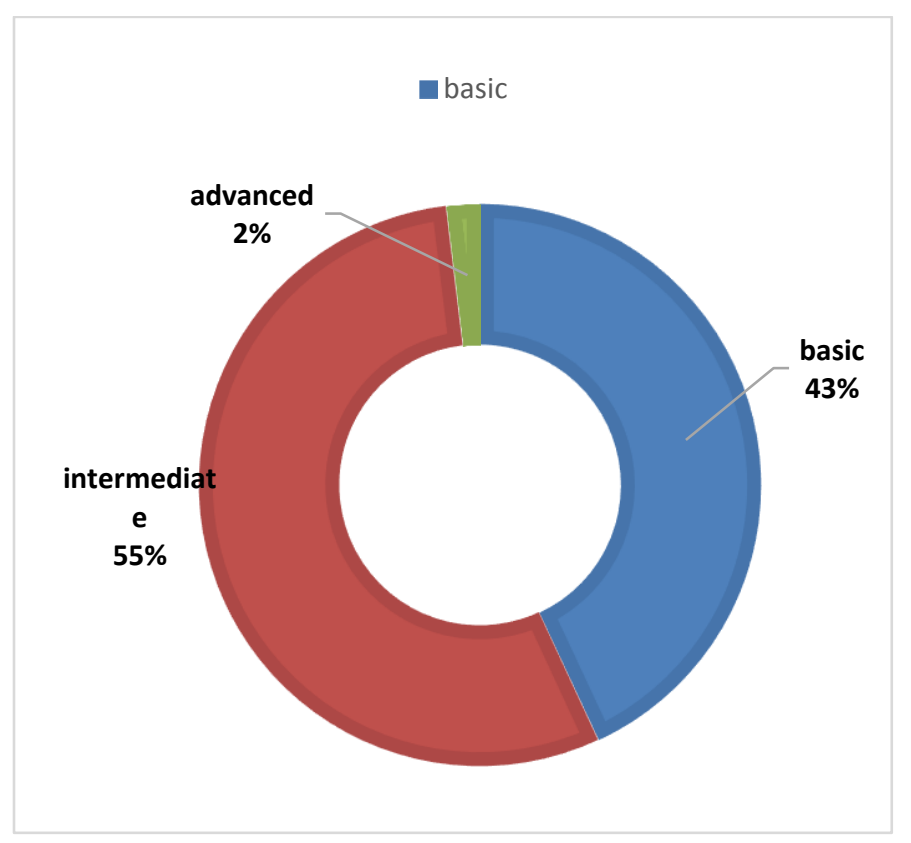

As demonstrated in Figure 3, the comparison between the three channels of communication demonstrates the dominance of basic-level questions in the reference transactions conducted via text messaging. The email questions show a close to even split between intermediate and basic questions, while the transactions conducted via chat, surprisingly, tend to include more intermediate-level questions than those conducted via email.

The distribution by the level of complexity of transactions conducted via chat and email was compared with results from the previous study. In the previous study, the split between intermediate and basic levels was 50\%/50\%, while the current results indicate a slight increase of the number of intermediate-level questions with 55\% intermediate questions and $43 \%$ basic, suggesting that for chat and email overall, questions asked have become more complex. This finding confirms the anecdotal evidence of librarians at McGill that that chat and email questions have become more complex over time. 
Figure 3. Question Level: Text vs. Chat vs. Email

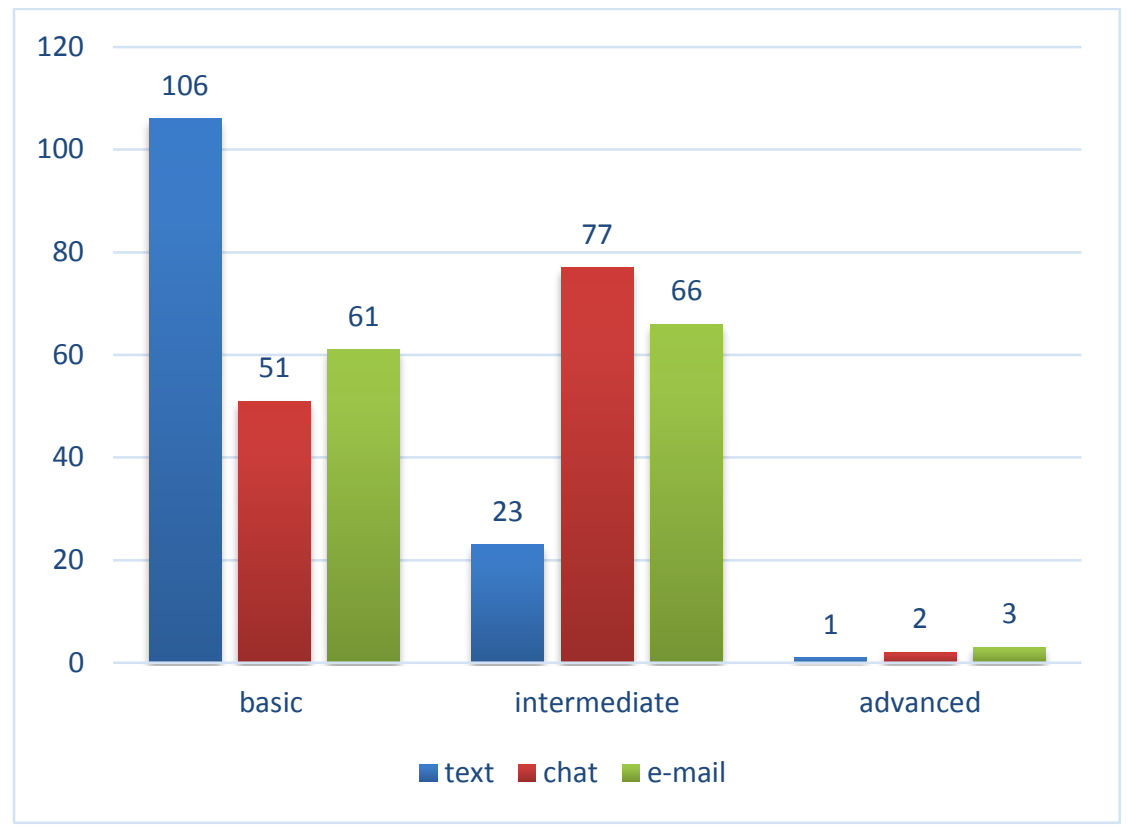

\section{Question Type/topic}

The results show that users gravitate toward asking questions of a certain type (on certain topics) through one channel of communication rather than another (see Figures 4 and 5).

\section{Figure 4. Question Type/Topic: Text}

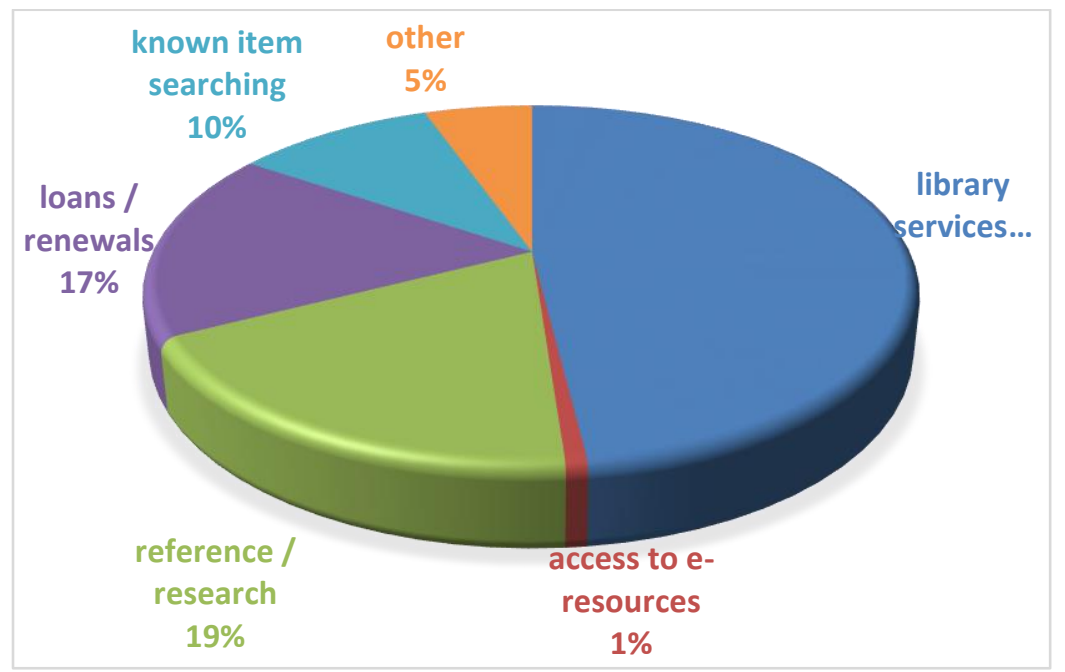


Figure 5. Question Type/Topic: Chat \& Email

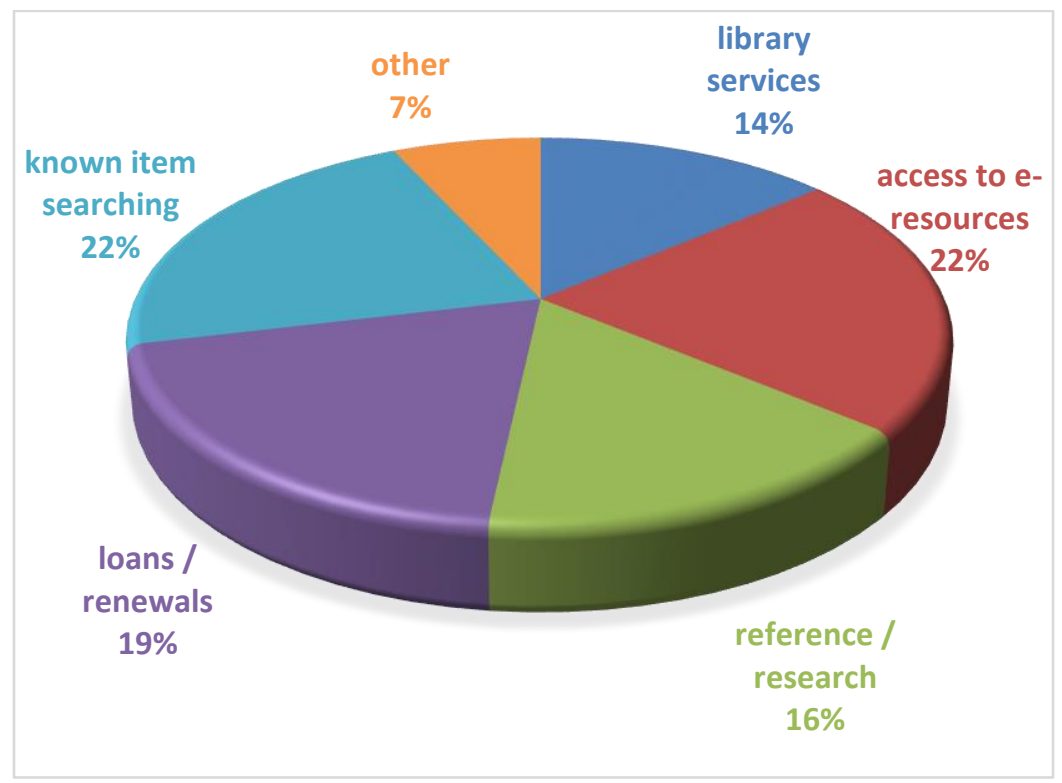

When the distribution of question types is compared between texting and chat/email, there is a notable dichotomy in users' choice of communication channel. In the studied sample, questions of the type that can be described as pertaining to library resources, such as "known-item-searching" and "access to e-resources," are noticeably more frequent in chat/email (44\% of the overall number) than in texting (only 11\%). Conversely, $48 \%$ of queries sent via texting are related to services (when combining hours, policies, and general/other library services) as opposed to only $14 \%$ of those sent through chat/email. The most popular question type sent via texting was "library services" accounting for $48 \%$ of questions, while for chat/email, there was a tie between "access to e-resources" and "known-item searching," each accounting for $22 \%$ of the total. It is worth noting that in the studied sample, the percentages of reference/research and loans/renewals queries were comparable in both texting and email/chat. 
Figure 6. Question Type/Topic: Text with Services Subdivided

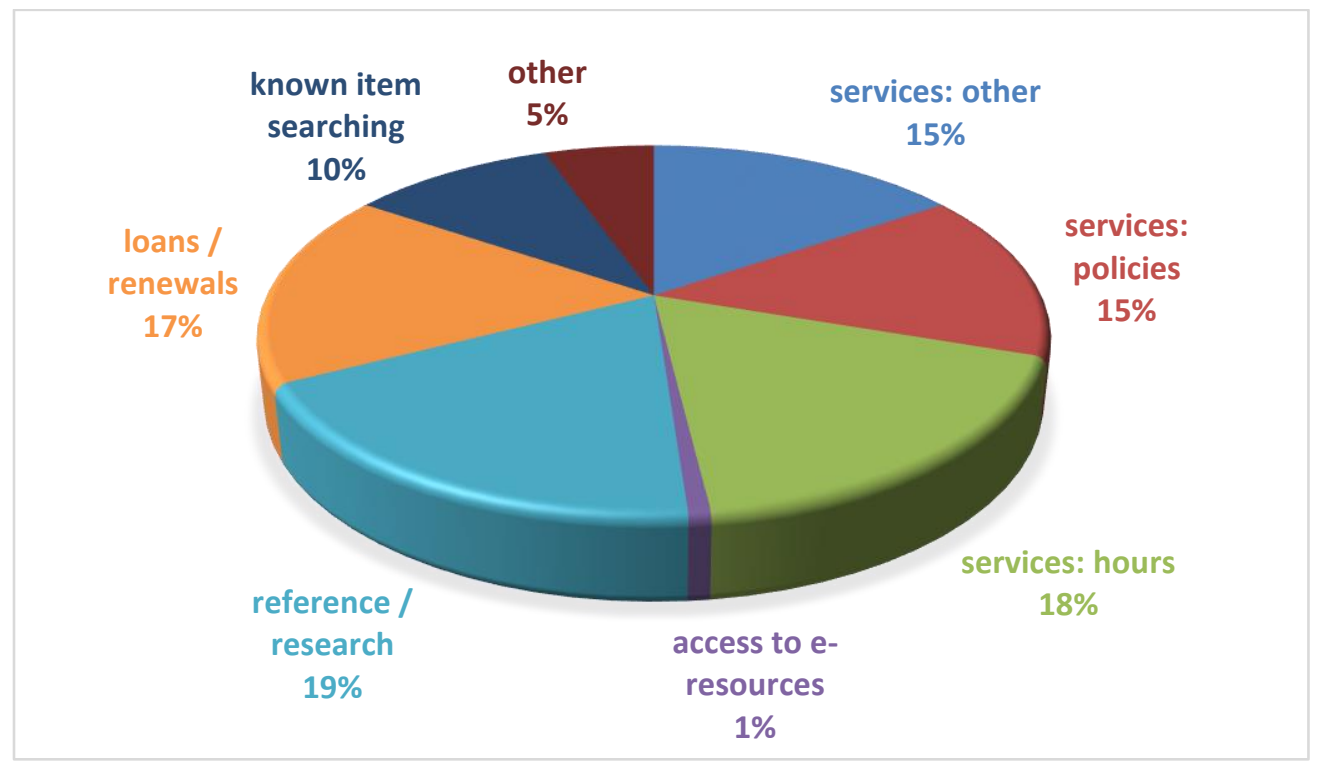

Figure 7. Question Type/Topic: Chat \& Email with Services Subdivided

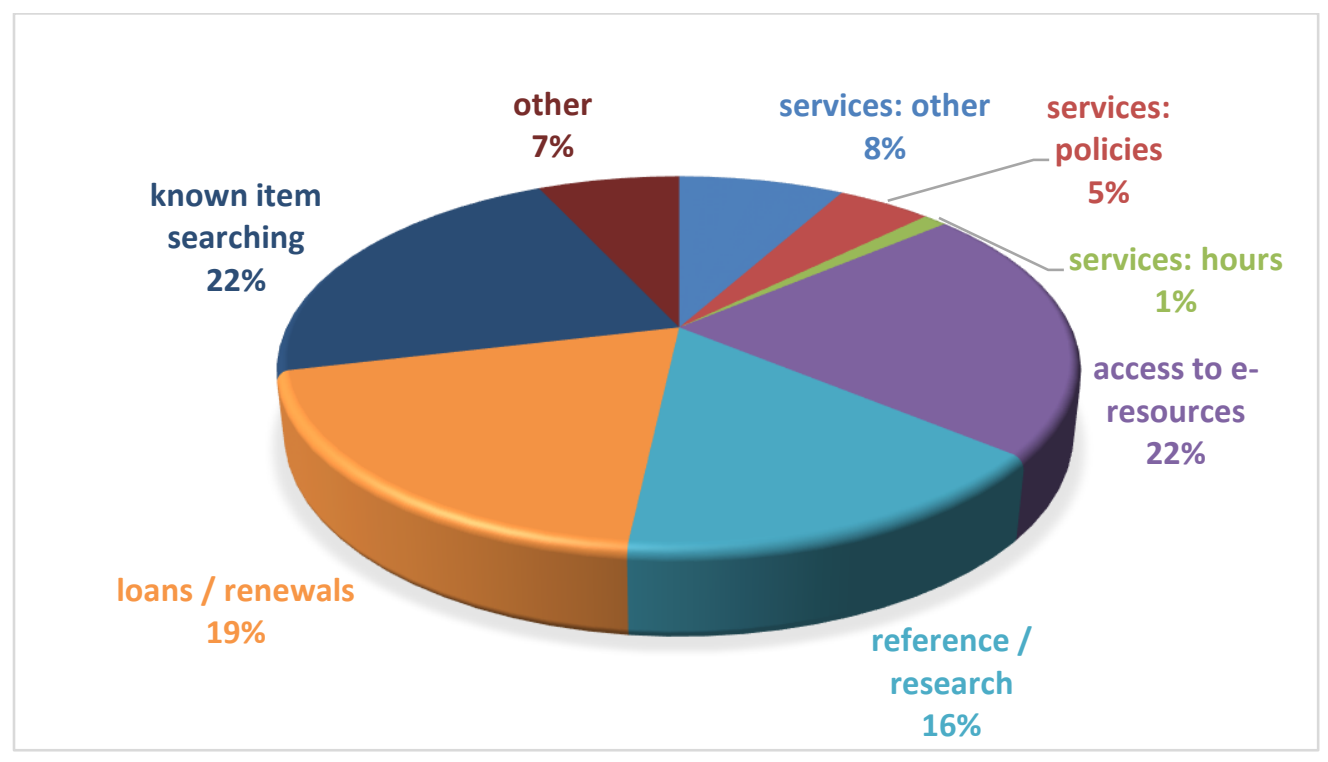

A further breakdown by the type/topic shows a significant difference in two types of servicerelated questions especially: queries about opening hours and library policies. While $18 \%$ of the studied sample of questions sent via text were asked on the subject of opening hours, in the queries received via chat/email, the category accounted for only $1 \%$ of the total number. Similarly, 
questions about library service policies were more frequent in text messages that in chats/email ( $15 \%$ vs. $5 \%$ of the studied sample) (see Figures 6 and 7 ).

In comparison with the previous study, some interesting patterns emerge. The distribution by question topic/type was remarkably similar. Categories that did not show any marked differences are: "known-item searching" (22\% of the total in both studies), "loans/renewals" (18\% in the past study vs. $19 \%$ in the current one), "reference/research" (17\% vs. 16\%) and "library services" (13\% vs. 14\%). The number of uncategorized questions (called "other") decreased from the previous study (at $14 \%$ ) to the current one (7\%). In contrast, the number of questions related to "access to e-resources" notably increased from $16 \%$ in 2014 to $22 \%$ in $2016-2017$.

\section{Referrals}

It is worth noting that the percentage of questions referred to another unit or librarian is almost double in chat/email queries as compared to the questions sent via text messaging, $27 \%$ vs. $10 \%$. The number of referrals for chat/email questions also increased in comparison with the 2014 transactions analyzed in the previous study, when it accounted for only $17 \%$ of the sample.

\section{Discussion/Conclusion}

\section{Question Level and Type/topic}

Findings of this study show that users will gravitate toward asking certain type of questions in one venue rather than another and that the type of queries most often asked via texting are basic. These tend to be more service-related such as hours and policies and less about specific electronic resources. Considering the "keeping it short" nature of texting interactions, the authors were not surprised that users gravitated toward quick-answer, less complex types of questions in the texting environment, as opposed to the other modes of communication offered by the virtual reference service, namely email and chat. This behaviour cannot be attributed to any influence from the library, given that no specific instructions are provided to users pertaining to what types of questions they should direct to which method of communication. These findings concur with some of the previous literature and suggest that a different type of response is appropriate to user queries within texting in comparison with chat/email. The study findings indicate that the texting service is generating different levels and kinds of questions than traditional virtual reference, suggesting that the new channel of communication fills a different information need. Given the different types 
of questions conducted via text, the service appears to be filling a complimentary role in relation to other traditional virtual reference communication channels used within libraries. This finding concurs with previous literature, which suggests that texting "is not cannibalizing users from chat and email services" (Vardeman \& Barba, 2014, p. 175). This is a good case for more libraries to offer this service.

The guidelines provided to staff at McGill Library have been to treat text messaging somewhat differently from chat and email, mostly due to current limitations not allowing users' messages to thread when they arrive into the library's virtual reference software. Library staff have been encouraged to answer basic user queries within the texting environment and take more complicated transactions out of texting and into other mediums. Given what the current study has shown us (that simple queries within the texting being most prevalent), the policy seems apt (and will remain in place for the time being).

However, simply because the results indicate that users are currently asking quick-answer type questions does not necessarily suggest that users are averse to longer, more complex interactions via texting. The study's findings show that texting tends to be shorter interactions on less complex topics but as cellphones and other mobile devices become the new normal for many types of tasks traditionally carried out on desktop or laptop computers, in the near future, there may be less and less of a need to treat chat, text and, email interactions differently from one another.

Not only do the study findings show that question levels between text and chat/email is markedly different, it also shows that there are notable differences in question type. As noted earlier, for example, $18 \%$ of text transactions pertained to opening hours, whereas in via chat/email transactions, the category accounted for only $1 \%$ of the total number. This difference could be explained by differences in locations (i.e. in front of a computer in the case of chat/email vs. elsewhere without ready access to a computer in the case of texting). As Gervasio mentions, questions in a texting environment often derive from different locations (2012, p. 46), where ready access to the library website, with details about hours, is not necessarily as easy to access. 
Conversely, certain types of questions were more prevalent in chat/email than in texting, such as "known-item-searching" and "access to e-resources." This discrepancy could equally be explained by once again considering context. This could also be due to the same phenomenon described by Gervasio where users of text are often seated elsewhere other than in front of a computer. These types of queries usually originate when users encounter difficulties in a search of the library's catalogue, a situation that may not be occurring as frequently when users are texting from their cell phones. At present, users may be accessing the library catalogue and many of the library's databases from their cell phones less frequently than from laptops or desktops, given that many resources are not yet very mobile-friendly. The findings from the current study differ from what Cornell librarians reported when they noted: "It is interesting and significant that we get quite a few texts about online resources. Even when users are sitting at a computer, some users prefer to use their cell phone to get help, rather than clicking on the Ask a Librarian web page" (Cole \& Krkoska, 2011, p. 8). It is difficult to account for these differences in question type within texting from one institution to another and may require further investigation.

Another interesting finding related to question type is that in the studied sample, the percentage of "reference/research" transactions, albeit low, was comparable in texting and in chat/email. Previous literature suggests that users are not averse to using texting for complicated queries (Cole $\&$ Krkoska, 2011, p. 7). Perhaps, under certain circumstances, users may prefer texting even for more complex interactions.

\section{Referrals}

The percentage of referrals in texting vs. chat/email was very different, with $27 \%$ referrals in chat/email and $10 \%$ in texting, while loans-related questions (often a source of referral) were about the same. These findings concur with Breitbach and Preito who state that not much referral is taking place in texting because the questions tend to be basic (2012, p. 194). Perhaps this lower percentage is also partly due to differences in question type, namely that more questions about eresources take place via chat/email (and often lead to referrals to collection services staff).

The current study saw an increase in the percentage of question referrals from the previous study where $17 \%$ of questions were referred. Perhaps this is due to increases in the number of 
questions related to access to e-resources (the share of e-resources-related questions increased significantly since the last study). The library has also increased the overall numbers of e-resources available through the library and often, there are questions related to their access and user. Another explanation for the increased number of referrals since the earlier study could be due to a noticeable increase in question difficulty (with the number of questions in the intermediate category having increased), perhaps requiring that librarians with specific subject specialization are needed to reply.

\section{Texting as a Different Type of Virtual Reference Service}

The overall low number of texts is difficult to explain. From the findings, it seems that texting fills a different need from other virtual reference methods and could likely benefit from either increased promotion via different methods than other virtual reference. As Gervasio says: "the near-synchronicity, anonymity, and brevity of SMS set it apart from other virtual communication, but the defining characteristic of SMS is its mobility" (2014, p. 55). She urges libraries to consider the "physicality" of texting, "unlike chat and email, texting is not a purely virtual experience. Texting can be immediate, personal, and influenced by a range of real-world phenomena" (2014, p. 55). Libraries should consider this fundamental difference in the way the service is advertised and accessed. It makes sense that the way users access the library's chat/email service is via the library website because users are already in front of a computer, but with texting, there is not as much logic in advertising it on the website. Perhaps better would be to promote it in physical locations where students might be with their phones but not necessarily with a laptop in front of them. For example, Gervasio’s study led to her library promoting the texting service from physical locations near printers, quiet study zones, and in the stacks $(2014$, p. 56). It is clear that texting is not the same kind of medium as chat and email.

People often consider texting to be a very personal mode of communication, something they use for one-on-one communication. Texting has a privileged and coveted place in a person's dayto-day life given that it is mostly used for contact with friends and family. Kohl and Keating understood the nature of texting as a personal means of communication and their goal in launching their texting service was to render the library's text a librarian contact information "a vital number to add to [a user's] speed dial" (2009, p. 106). If the library's texting contacts are in someone's 
phone, they are there among privileged contacts. The personal nature of texting has implications for both service promotion and delivery. Texting is indeed a different type of virtual reference service. This is a factor to be considered in how the service is delivered.

\section{Limitations of Current Study}

One limitation of the current study is the small number of texts that were analyzed. This was the first year that the service was offered, which could partially explain the low use. However, this experience also concurs with previous findings that report low use of texting (Vardeman \& Barba, 2014, p. 178; Breitbach \& Prieto, 2012, p. 193). Also, the total number of texts and chats/emails received differ largely from each other with 324 texts and 8,779 chats/emails received during the sampling period. To mitigate this issue, texts were compared only to a sample of chats/emails selected using systematic random sampling.

Another limitation of the current study was the problem with extracting the chat transactions from the QuestionPoint software, resulting in only part of the total number of transactions being sampled, 3,807 out of 5,392. However, this is unlikely to have affected findings since the total number of chat transactions extracted was still very high ( $70 \%$ of the total) and covered the entire one-year corresponding to the sample of texting transactions.

\section{Implications for Practice}

The study has several implications for delivery of texting service. Important to note is the type of questions often asked via text. Considering the prominence of questions related to hours and library/services policies received via text, it would be helpful to increase the visibility of these types of information on the library mobile site.

Another implication is the need for additional and different types of promotion of the texting service. As previous research indicates, texting does not enjoy the same level of popularity as other forms of virtual reference, which is confirmed by the authors' local experience. As noted with the McGill Library virtual statistics mentioned earlier, the chat/email service has grown over the years. It takes time to build a clientele with any form of reference. Especially in the context of a texting service, not only do users need to be made aware of the service, but also texting gives the library 
a personal point of contact with users and a place inside their personal space, their cell phone. Promotion of texting may require a different approach with a focus on physical spaces, inside and beyond the library, considering that users might be using the service while not seated in front of a computer. Publicity methods could focus on the presence of information about the service in physical spaces on campus (e.g. posters, digital signage screens, etc.). After additional promotional efforts have been carried out, the viability of the texting service could be reassessed.

The methods developed for the project can be easily adapted and applied for assessing and evaluating questions received by a virtual reference service in any type of library. It is a worthwhile exercise to examine question types in a given library since this surely differs from one library to another. The findings of this study can also help to better prepare reference providers for the demand for this type of service in the future.

\section{Future Research}

The current study points to several areas of future research. First, the question of whether users are gravitating toward other virtual reference services due to inherent difficulties with the medium, lack of awareness, reluctance to have the library inside their personal spaces or other reasons should be investigated. An interesting area of future research could be to conduct qualitative analysis via in-person interviews and/or focus groups of user preference for chat, text, and email, and also to further investigate in what context and for what reasons users choose a medium to contact the library, be it email, text or chat. It would be useful to conduct a qualitative investigation through interviews to determine what makes users choose texting over chat, especially in the context of cell phones becomingly primarily smartphones equipped with the internet (where chat services can also be utilized). This future study could also investigate in what types of circumstances, if any, users would find it advantageous to conduct an advanced query via text.

Future research could shed additional light on whether or not texting should be treated differently from other methods of virtual reference and whether or not a "keeping it short" or a "venue-blind" approach to texting, as advocated by Pearce et al., as described earlier, better serves user needs. Also, recent research suggests that although hyperlinks may facilitate informationsharing, they can simultaneously jeopardize the interaction with the user in certain contexts 
(Stommel, Paulus, \& Atkins, 2017, p. 65). It is implied that these types of short answers, consisting of hyperlinks, may alienate users. More research is needed, especially from the user perspective, on the subject of how the difference in methods of communication may influence users' perception of the service.

\section{Acknowledgements}

The authors are grateful to their colleague, Jill Boruff for reading and commenting on the draft of this article.

\section{Cited References}

Breitbach, W., \& Prieto, A. G. (2012). Text reference via Google Voice: A pilot study. Library Review, 61(3), 188-198. doi:10.1108/00242531211259319

Charlton, G. (2013, November 25). Consumers prefer live chat for customer service: stats. Retrieved from https://econsultancy.com/blog/63867-consumers-prefer-live-chat-forcustomer-service-stats/

Chow, A. S., \& Croxton, R. A. (2012). Information-seeking behavior and reference medium preferences: Differences between faculty, staff, and students. Reference and User Services Quarterly, 51(3), 246-262. doi:10.5860/rusq.51n3.246

Chow, A. S., \& Croxton, R. A. (2014). A usability evaluation of academic virtual reference services. College \& Research Libraries, 75(3), 309-361. doi:10.5860/crl13-408

Cole, V., \& Krkoska, B. B. (2011). Launching a text a librarian service: Cornell's preliminary experiences. The Reference Librarian, 52(1-2), 3-8. doi:10.1080/02763877.2011.521881

Collard, S., Whatley, K., \& Pearce, A. (2011, March). Reference philosophy in a mobile world: Evidence for service provision and sustainability. Paper presented at the Association for College and Research Libraries (ACRL) Conference, Philadelphia, PA. Retrieved from http://www.ala.org/acrl/sites/ala.org.acrl/files/content/conferences/confsandpreconfs/nati onal/2011/papers/reference_philosophy.pdf

Côté, M., Kochkina, S., \& Mawhinney, T. (2016). Do you want to chat? Reevaluating organization of virtual reference service at an academic library. Reference and User Services Quarterly, 56(1), 36-46. doi:10.5860/rusq.56n1.36 
Gervasio, D. I. (2014). Redefining virtual: Leveraging mobile librarians for SMS reference. International Journal of Digital Library Systems (IJDLS), 4(2), 44-69. doi:10.4018/IJDLS.2014070104

Kohl, L., \& Keating, M. (2009). A phone of one's own: Texting at the Bryant University reference desk. College \& Research Libraries News, 70(2), 104-118. doi:10.5860/crln.70.2.8128

Luo, L. (2011). Text reference service: Delivery, characteristics, and best practices. Reference Services Review, 39(3), 482-496. doi:10.1108/00907321111161449

Matteson, M. L., Salamon, J., \& Brewster, L. (2011). A systematic review of research on live chat service. Reference and User Services Quarterly, 51(2), 172-190. doi:10.5860/rusq.51n2.172

McLuhan, M. (1964). Understanding media: The extensions of man. New York: McGraw-Hill. Pearce, A. (2010). Text message reference at NYU libraries. The Reference Librarian, 51(4), 256-263. doi:10.1080/02763877.2010.503314

Pearce, A., Collard, S., \& Whatley, K. (2010a). Say what? An SMS transcript analysis at New York University. In M. Ally \& G. Needham (Eds.), M-libraries 2: A virtual library in everyone's pocket (pp. 255-264). London: Facet.

Pearce, A., Collard, S., \& Whatley, K. (2010b). SMS reference: Myths, markers, and modalities. Reference Services Review, 38(2), 250-263. doi:10.1108/00907321011045016

Peters, T. A. (2010). Left to their own devices: The future of reference services on personal, portable information, communication, and entertainment devices. The Reference Librarian, 52(1-2), 88-97. doi:10.1080/02763877.2011.520110

Pew Research Center. (2015, April). U.S. smartphone use in 2015. Retrieved from http://www.pewinternet.org/2015/04/01/us-smartphone-use-in-2015/

Ruppel, M., \& Vecchione, A. (2012). "It's research made easier!” SMS and chat reference perceptions. Reference Services Review, 40(3), 423-448. doi:10.1108/00907321211254689

Stommel, W., Paulus, T. M., \& Atkins, D. P. (2017). "Here's the link": Hyperlinking in servicefocused chat interaction. Journal of Pragmatics, 115, 56-67. doi:10.1016/j.pragma.2017.02.009

Vardeman, K. K., \& Barba, I. (2014). Reference in 160 characters or less: The role of text 
messaging in virtual reference services. Internet Reference Services Quarterly, 19(3-4), 163-179. doi:10.1080/10875301.2014.984098

Vecchione, A., \& Ruppel, M. (2012). Reference is neither here nor there: A snapshot of SMS

reference services. Reference Librarian, 53(4), 355-372. doi:10.1080/02763877.2012.704569

\section{Appendix 1: Question Coding Scheme}

1. Text, Chat, E-mail

2. Referred: $\mathrm{Y} / \mathrm{N}$

3. Level of questions:

- basic: responds to a simple question using library information sources (catalogue, website, ready reference)

- intermediate: assists users with intermediate-level questions or support, may require use of several information sources, and often involves user instruction

- advanced: responds to a user's question using advanced expertise in the service area. Interactions are often multi-faceted and/or inter-disciplinary and subject specialists may need to be consulted.

4. Theme of questions

- Library services: policies

○ Library services: hours

- Library services: other

- Issue with access to e-resources

○ Reference/ research

- Loans/ renewals

○ Known item searching

○ Other 